\title{
Uncovering the Concentration Phenomenon of Vehicle Mobility in Urban Areas
}

\author{
Zhi Yu${ }^{1, *}$, Bing Zou ${ }^{1}$, Zhaocheng He ${ }^{1}$, Min Huang ${ }^{1}$, Kaiying Chen ${ }^{2}$, and Liangkui Luo ${ }^{1}$ \\ ${ }^{1}$ Guangdong Provincial Key Laboratory of Intelligent Transportation Systems, Research Center of Intelligent \\ Transportation System, Sun Yat-Sen University, Guangzhou, Guangdong 510006, China \\ ${ }^{2}$ Guangdong Fundway Technology CO., Ltd, Guangzhou, Guangdong 510006, China \\ *zoub8@mail2.sysu.edu.cn \\ +these authors contributed equally to this work
}

\begin{abstract}
Extracting the regularity of individual vehicle mobility has long been a general research topic that has numerous applications for urban management and services. In this paper, we combine vehicle mobility information extracted from vehicle identification data with a large and directed road network, to understand vehicle mobility and how it shapes the road usage profile in an urban city. We propose here a versatile method to extract the division point of vehicle mobility distribution, which is designed to observe the interesting phenomenon whereby, similar to the inequality in wealth distribution in economics, surprisingly few vehicles contribute to the larger proportion of road usage. Based on the relationship between vehicle mobility and urban roads, we reveal road usage profile (e.g. central, transit, branch, local), depending on traffic flow and vehicle mobility inequality features, in contrast to traditional topology indicators and aggregated indicators. We also validate for the first time a methodology which uncovers the road usage characteristics from the microscopic perspective of vehicles. These results allow us to create a quantitative strategy to note the few but decisive vehicles that add to traffic congestion and to put into perspective the transition from traditional aggregated approaches to individual-based practices in transportation research.
\end{abstract}

\section{Introduction}

Recent data sources have opened up amazing possibilities for quantitative research that focuses on individual mobility patterns and urban dynamics, such as allometric scaling relationships ${ }^{1}$ and morphological comparison of cities, enabling a new scientific urban theory ${ }^{2}$. An unprecedented volume of data has been used, including smart card data ${ }^{3}$, mobile data ${ }^{4-7}$, car GPS $^{8}$, and some check-in data from Internet sites such as Twitter ${ }^{9}$ and Facebook ${ }^{10}$. Meanwhile, many alternative models have been conceived, aimed at uncovering human scaling ${ }^{1,11-17}$ and detection of urban land usage based on human interactions ${ }^{5,18}$. Additionally, many structural indicators shared by complex systems, such as centrality and node degree ${ }^{19-21}$, have been used to hint at the universal process of urban traffic systems, and thus at the possibility of deconstructing cities.

Extracting useful information from these huge datasets has become a fundamental problem that requires solving. Generally, we are always interested in providing real insight into the phenomena that occur in cities and in singling out the essence of a few such phenomena that govern urban dynamics from multi-scale information. Uncovering the existence of a variety of processes helps us to tackle systemic challenges as well as to lay the groundwork for comparing different systems. In fact, despite the numerous methods ${ }^{22-24}$ used to capture and understand observed empirical regularities, no exploration has yet been conducted of how mobility shapes road usage at the individual level.

Several possible methods to explore individual mobility patterns and human scaling law have been proposed in the literature using high spatial and temporal resolution data. These studies aim to develop models of universal processes in human mobility, such as commuting patterns ${ }^{3,4}$, daily mobility motifs ${ }^{25}$, and detection of mobility pattern changes ${ }^{26}$. Apart from these, to uncover the scaling law underlying human mobility, a wide variety of data-based statistical descriptions ${ }^{13}$ (Lévy flights, long-tail distribution) and mathematical models ${ }^{14-17}$ (gravity model, random walk model, radiation model) have been derived at a quantitative level. Based on these models, a theoretical understanding of empirical individual behaviours has been achieved ${ }^{27-30}$. A remarkable breakthrough has recently occurred in the field of quantitative cities or roads, due to the availability of high-resolution spatial-temporal data. This breakthrough represents a step towards a theoretical understanding of observed phenomena, combining the individual behaviour of quantities with the spatial mobility network. With the insights into individual mobility, many methods related to quantitative geography and traffic congestion that take scaling law into account have been further conceived. These methods use these data to understand the mechanism of traffic congestion ${ }^{23}$ and how human mobility shapes cities ${ }^{22}$, including activity centres ${ }^{7}$, space density ${ }^{31}$, road usage patterns ${ }^{24}$, and urban spatial structure ${ }^{6,32}$. Thus 
far, most such methods have been based on topology indicators ${ }^{19,21,33}$ (landscape density, betweenness or degree of linking, connectivity, etc.) and multiple aggregated indicators (travel time, OD matrix ${ }^{6}$, hot spots ${ }^{7}$, etc.). In so doing, they extract road usage or city spatial structure from large-scale crowd flows. In previous traffic planning, which was based on individual traffic travel survey data, census data, and socio-economic indices in cities, urban planners and architects could estimate trip density and distribution patterns ${ }^{31,34}$, and then picture the schematic road networks, including land functions and road hierarchy.

The main obstacle to thoroughly deconstructing traffic systems from the essential features of traffic operation resides in the lack of adequate data and rough granularity considering temporal resolution. Moreover, the analysis remains limited to sample-based and aggregated approaches, rather than considering individual differences. Sampling assumptions in traditional models such as the four-step method often suppose that the behaviours of different individuals are consistent with each other. However, if a small part of a sample is used to estimate the overall distribution law by aggregation, it will cause some real distribution at the individual level to be mistakenly concealed or distorted. In particular, cities are not only a collection of various functional areas including commercial areas, homes, and workplaces but also the carriers of individual mobility and life. To use an expressive metaphor, a city is like a living body, while an individual traveller is akin to its DNA-the minimum basic unit which determines the characteristics of the life body. Individuals with different specific identities demonstrate large differences in mobility or activities due to factors such as travel motivation, attributes, and choices. From this perspective, it is necessary to transform ideas from aggregate to individual by capturing the DNA of the traffic system and the accurate behaviours of its individual users.

Based on the identity of individual mobility data, some interesting questions can be explored from the perspective of micro-individual behaviours: Since we know where most individuals live and where they go, can we find out who is traveling? Which part of individual vehicles is consuming road resources in a city, contributing to traffic congestion? Does a small part of travel vehicles play a decisive role and what is their influence? How do these individuals influence urban road usage? How does individuals' mobility shape the spatial organisation, growth, and development of each city?

Automatic vehicle identification (AVI) data contain the identity information and real-time location of vehicles (see Supplementary 1.1). This paper is based on the vehicle mobility datasets obtained from the AVI system in Xuancheng City, Anhui Province, China. We selected vehicles as the research object to study individuals' behaviour and propose a simple and versatile method designed to note the interesting phenomenon whereby surprisingly few vehicles contribute to the larger proportion of road usage. In the next section, we will describe in detail the method used. Our results also allow us to provide quantitative insight into the relationship between vehicles and road usage. Finally, this framework allows us to determine road categories based on their actual usage characteristics in contrast to traditional topological and aggregated indicators, and to classify roads according to both their traffic flow and road usage concentration degree.

\section{Results}

\section{Average Division Point of vehicle mobility distribution}

In 1912, economists proposed using the Gini Coefficient to measure a country's disparity in income distribution, which helps determine the degree of concentration in wealth distribution. In fact, the Gini coefficient functions as a summary statistic and has been widely adopted for different regions and in different time periods.In this study, a similar phenomenon was found for urban vehicles' use of urban spatial and temporal resources, benefiting from the recent availability of pervasive data that tags vehicles' identity.

Suppose a city road segment is equipped with a detector that identifies a vehicle's ID and driving direction. When a time stamp is available, we can obtain historical data of any time scale and vehicle trip through the given road segment. For any given segment, we sort the vehicles by decreasing rank according to their trip frequency $\omega(x)$ and denote them by $\omega(1)>\omega(2)>\ldots>\omega(n)$, where $n$ is the trip frequency of the vehicle. Let $f(\omega)$ be the probability density function of trip distribution. Accordingly, $F(\omega)$ is the cumulative probability function of vehicle share, with a trip frequency of no less than $\omega$. The curve is constructed by plotting on the $\mathrm{x}$-axis the proportion of vehicles (as Fig1 $\mathrm{x}$-axis shows):

$$
F(\omega)=\int_{0}^{\omega} f(t) d t
$$

and on the y-axis the corresponding proportion of vehicles' trips $L$ with:

$$
L(\omega)=\frac{1}{\mu} \int_{0}^{\omega} t f(t) d t
$$

$\mu$ is the average trip frequency of vehicles for a given segment:

$$
\mu=\int_{0}^{+\infty} t d F(t)
$$


If we let $p=F(\omega)$, the curve can be written directly as:

$$
L(p)=\frac{1}{\mu} \int_{0}^{p} F^{-1}(t) d t
$$

Of these, the horizontal axis represents the cumulative percentage of vehicles, while the vertical axis represents the percentage of vehicles traveling, as Fig1 shows.

If each vehicle has a similar or even identical trip frequency, the curve will become a diagonal from $(0,0)$ to $(1,1)$. Thus, it always remains above or equal to the $45^{\circ}$ line. In fact, $L(p)$ is an increasing convex function of $\mathrm{p}$. Its first derivative is always positive(as shown in the following formula).

$$
\frac{d L(p)}{d p}=\frac{F^{-1}(p)}{\mu}
$$

We find that when the tangent of the curve is parallel to the 45-degree diagonal, the point $\mathrm{M}$ with a slope of 1 can actually determine the general shape of the curve. The rate of increase of the curve to the left of this point exceeds that to the right of the point with a uniform increase of the $\mathrm{x}$-axis. On the curve, the more leftward this point is, the greater the degree of curve bending, the stronger the vehicle mobility inequality, and, intuitively, the more concentrated the travel resources(e.g.trip frequency, trip time, trip length) in a small number of vehicles. This observation inspired us to construct a criterion to distinguish between the small proportion of vehicles that occupy the larger proportion of travel resources and the larger proportion of vehicles that consume few travel resources.

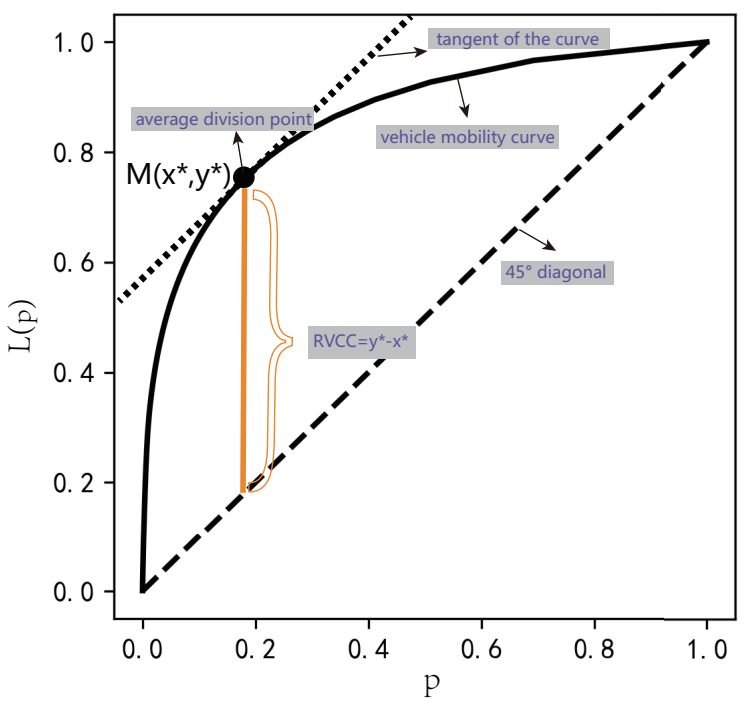

Figure 1. Principle diagram of vehicle mobility curve and average division point

When the tangent of the curve is parallel to $45^{\circ}$ diagonal, that is, let the first derivative of $L(p)$ be 1 , we can obtain $p=F(\mu)$ or $\omega=\mu$. The point with a slope of 1 is actually coincident with the average frequency of the vehicle's trip. Thus, we define the average division point $\left(x^{*}, y^{*}\right)$ on the curve by the following equation:

$$
\left\{\begin{array}{l}
x^{*}=F(\mu) \\
y^{*}=\frac{1}{\mu} \int_{0}^{F(\mu)} F^{-1}(t) d t
\end{array}\right.
$$

Unlike some summary indexes such as the Gini coefficient, the average division point on the curve can not only reflect inequality but also clearly demonstrate which part of the total vehicles is the dominant cause of inequality and its specific contribution to that inequality. It describes more information about the profile of vehicle mobility distribution. The specific difference is further described in Supplementary Figures S7 and S8. The average division point is not only the demarcation point of dominant and secondary travel vehicles but also a clear expression of the degree of travel sources they represent. $X^{*}$ $=\left\{x_{1}^{*}, x_{2}^{*}, x_{3}^{*}, \ldots x_{n}^{*}\right\}$ represents the proportion of all vehicles that have travelled more than the average frequency of trips in a particular road segment. $\boldsymbol{Y}^{*}=\left\{y_{1}^{*}, y_{2}^{*}, y_{3}^{*}, \ldots y_{n}^{*}\right\}$ is the relative frequency of total trips held by all vehicles who travelled more than the average frequency. This helps us to pinpoint the dominant vehicles using a specific road segment that need to be focused on and managed. 


\section{Vehicle identity data, road usage concentration coefficient, and vehicle sources of a road segment}

Large-scale individual mobility data were extracted from multi-source data in the city, such as GPS, mobile data, or social check-in data. With the advancement of image recognition technology, AVI systems such as License plate recognition, RFID, and automotive electronic identification have been widely applied, particularly in Asian cities such as those in China, allowing vehicle mobility information to be obtained. Compared to traditional section and mobile detectors, AVI systems are an efficient way to provide lane-based and real-time vehicle location data. Every record from an AVI system contains vehicle identity(license plate number), detector ID, lane number, and timestamp(see Supplementary 1.1: Vehicle Identity detection). In the following, we apply the vehicle mobility curve and average division point extraction method to urban vehicle mobility data that have been extracted from AVI systems in Xuancheng City, Anhui Province, China, over a 1-year period(see Methods section for details on the dataset). We divided the city into 218 traffic zones and digitised all directed segments in the studied urban area, providing the basis to analyse road segments and vehicles' mobility including their origins and destinations(ODs). The favourable vehicle-level data environment of Xuancheng city enabled us to understand the origins of the vehicles in each road segment and encode the essence of urban traffic systems.

Based on the AVI system data, taking a road segment as an example, we can extract all the vehicles passing through that road segment and the traffic flow produced by the vehicle during a given time period. Then, following the above methods, we can sort the vehicles in decreasing rank according to their contribution to traffic flow. Thus, vehicle trip frequency is characterised by the vehicle mobility curve $L(p)$. We also calculated the average frequency of trips of all vehicles and put this average into $L(p)$ to find the horizontal and vertical axes, that is, the average division point of the vehicle mobility curve $\left(x^{*}, y^{*}\right)$ (see Figure 1). The average division point informs us that for a long-term congested road segment, it is no longer difficult to target the surprisingly few vehicles and grasp the total amount of usage produced by this part of the total vehicles.
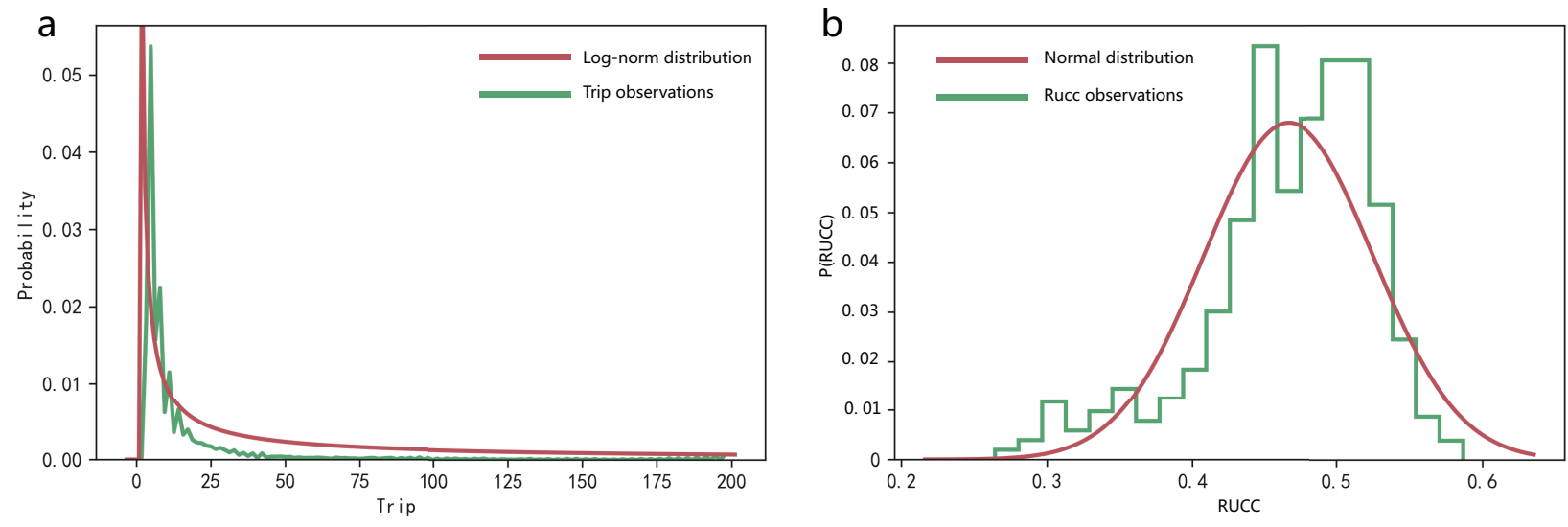

Figure 2. Distribution of vehicle trips on a certain road segment(Zhuangyuan South road) and RUCC(rare vehicle concentration coefficient) in August, 2018. (a)The vehicle trips on Zhuangyuan South road follow a log-norm distribution $P\left(K_{\text {trip }}\right)=\mathrm{e}^{-\left(\ln \left(K_{\text {trip }}\right)-\mu_{\text {trip }}\right)^{2} / 2 \sigma_{\text {trip }}^{2}} /\left(\sqrt{2 \pi} \sigma_{\text {trip }} K_{\text {trip }}\right)$ with $\mu_{\text {trip }}=0.81, \sigma_{\text {trip }}=1.01$. Zhuangyuan South road is a road segment in an urban central area with heavy traffic. (b)The degree distribution of RUCC for Xuancheng City in August 2018 is well approximated by a normal distribution $P\left(K_{\text {rucc }}\right)=\mathrm{e}^{-\left(K_{\text {rucc }}-\mu_{\text {rucc }}\right)^{2} / 2 \sigma_{\text {rucc }}^{2}} /\left(\sqrt{2 \pi} \sigma_{\text {rucc }}\right)$ with $\mu_{\text {rucc }}=0.46, \sigma_{\text {rucc }}=0.06$.

From this average division point, it is natural to calculate for each road segment a 'road usage concentration coefficient'(RUCC) defined as $y^{*}-x^{*}$, which comprises the concentration degree of the surprisingly few vehicles that, although low in proportion, contribute a large proportion of road usage. Conceptually, RUCC is actually a judge of vehicle mobility inequality degree and is here derived from the average division point. The greater the value of RUCC, the greater the concentration of vehicle mobility. The coefficient ranges from 0 to 1 as the distance between average division point and absolute average curve(see Figure 1). As Figure2 shows, the distribution of vehicle trips in Zhuangyuan South road(a typical example) is well fit by log-norm distribution. It displays the imbalance in vehicles' travel, which is what inspired us to define RUCC. In the next section, we present the applications of RUCC and combine it with road usage characteristics and comparison in urban areas.

The traditional failure to grasp vehicles' trajectory and ODs has limited the capture of massive vehicle sources related to roads' attractiveness in long-term usage. Here, we demonstrate that the proposed average division point provides an opportunity to pinpoint the surprisingly few vehicles on a specific road segment. We can essentially distinguish the few but decisive vehicles, that is the vehicles of $X^{*}$, and learn their influence according to their contribution to the traffic flow of each road segment, namely $\boldsymbol{Y}^{*}$. Furthermore, the average division point can be applied sequentially when faced with large numbers of vehicles. 
We defined the average division point of all vehicles $\left(x_{1}^{*}, y_{1}^{*}\right)$ first minority. Consequently, when facing the vehicles $x_{1}^{*}$, we performed the same descending sorting method according to their contribution to traffic and found the second average division point $\left(x_{2}^{*}, y_{2}^{*}\right)$, defined as second minority. Similarly, $\left(x_{3}^{*}, y_{3}^{*}\right)$ is third minority derived from the average division point of vehicles $x_{2}^{*} . P=\left\{\left(x_{1}^{*}, y_{1}^{*}\right),\left(x_{2}^{*}, y_{2}^{*}\right),\left(x_{3}^{*}, y_{3}^{*}\right), \ldots\left(x_{n}^{*}, y_{n}^{*}\right)\right\}$ is a cluster of average division point, which is designed to note the key vehicles sequentially and in depth. Taking a road segment as an example, as Figure 3 demonstrates, with the cluster $P$, we can identify that the average division points of the directed road segment are $\{(18.42 \%, 69.99 \%),(4.22,44.02 \%),(1.34 \%, 26.25 \%) \ldots\}$. This means that $69.99 \%$ of traffic flow of the directed road segment is contributed by $18.42 \%$ of the vehicles(first minority); $44.02 \%$ of the flow is contributed by $4.22 \%$ of the vehicles(second minority); and $26.25 \%$ of the flow is contributed by $1.34 \%$ of the vehicles (third minority). This indicates that the major usage of the road segment could be tracked to $18.42 \%, 4.22 \%$, and even $1.34 \%$ of all the vehicles (as shown in Figure 3). This allows us to continually trace back to the very few vehicles that contribute to traffic and respond flexibly to traffic origins at different usage degrees. Furthermore, we may explore the nature of these vehicles. Once our framework has identified the minority of vehicles, we can locate the major traffic zones of each road segment given the minority of vehicles' ODs(as Figure 3 shows). In so doing, we could depict the specific process of how the traffic flow of a road segment associated with specific vehicles is generated. This method also allows us to perform distinct analysis of peak and off-peak hours, or other periods. The specific results are listed in Supplementary Tables S5 and S6.

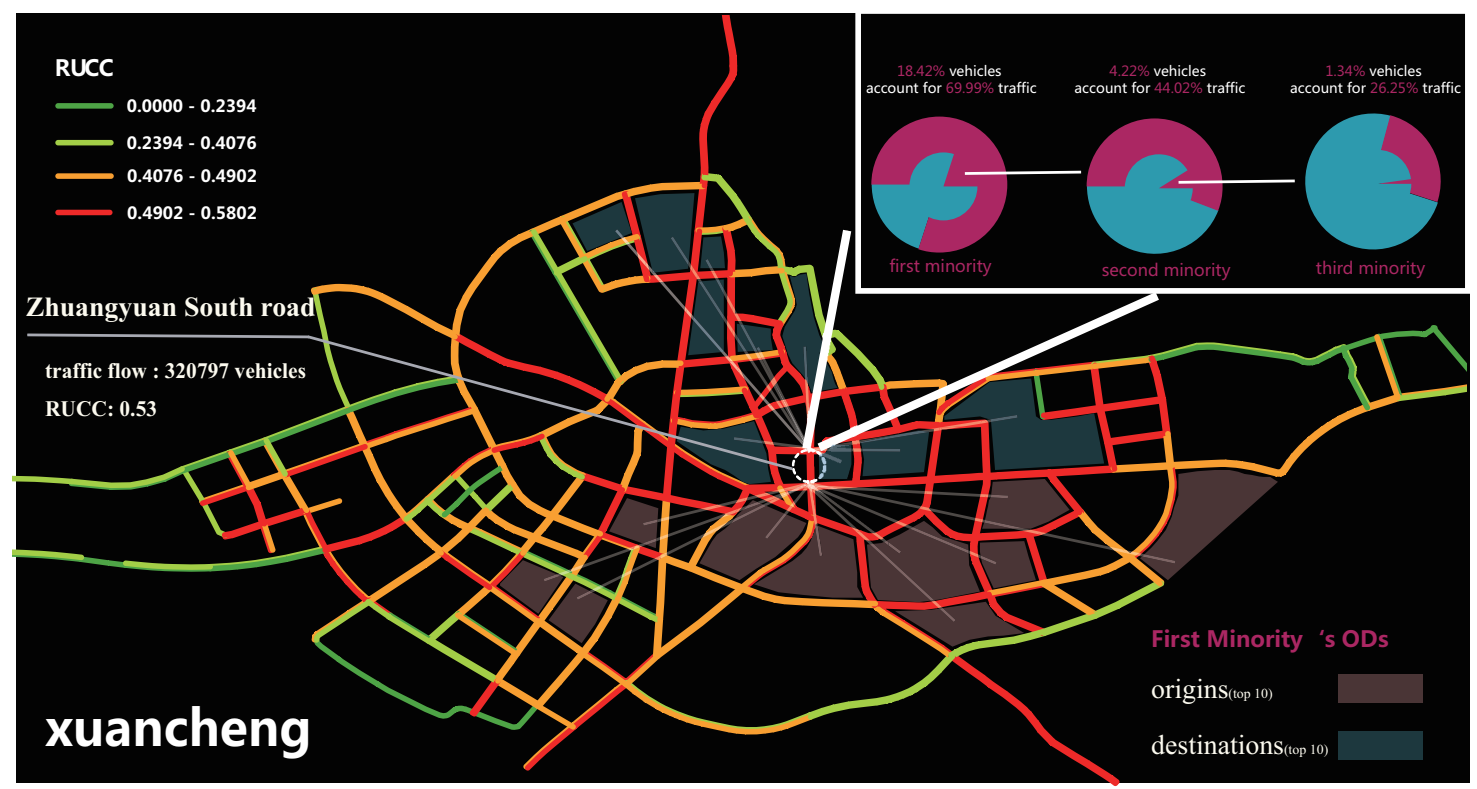

Figure 3. Encoding vehicle sources of road segments via average division point. (i) The colour of road segments represent their 'road usage concentration coefficient'(RUCC). Red represents a larger value and green a smaller. It is obvious that most road segments have a small RUCC whereas the central or peripheral road segments have a large RUCC. (ii) The pie chart on the top right represents from left to right the three minority vehicle sources in the selected road segments. Pink represents the decisive minority of vehicles, light blue shows the others; the inner rings represent vehicles $\left(x^{*}\right)$, while outer rings represent their influence $\left(y^{*}\right)$. (iii) Light red and light blue are the traffic zones of vehicles' origins and destinations in the selected road segment. The white lines are the links between associated zones and selected road segments.

Remarkably, our framework is not limited to the spatial and temporal scale. For example, for Xuancheng City, we obtained the vehicle mobility curves $L(p)$ of the city per day, week, month, quarter, and annual scale(see Supplementary Figure S9). We may observe that, as the time scale increases, the degree of curvature of the curve also increases, indicating that vehicles that are usually active in cities occupy increasing resources over time. This is analogous to the phenomenon of 'the rich get richer and the poor get poorer' in wealth distribution. The difference is that in urban transportation, this small 'rich' vehicle is determined by its operational nature and traffic demand. We note here that it would also be interesting to apply the method to different scales with different indicators. This would make it possible to obtain the vehicle mobility curve and average division point using our method for larger spatial scales such as regions, blocks, and even cities and for a wider range of temporal scales 
such as week, quarter, and even year. The indicators of vehicles' contribution can also further expand beyond traffic flow, to factors such as congestion, travel time, and driving distance.

\section{From Average Division Point to road usage classification}

Since the proposed average division point is a reflection of the relationship between vehicles and specific road usage, and a road segment's 'road usage concentration coefficient'(RUCC) quantifies the concentration degree of the vehicles using it, it again hints that RUCC can describe the characteristics of a road segment in the road network related to vehicle usage inequality. From the perspective of indicator selection, existing studies always extract the city usage pattern in an aggregated manner inspired by the large-scale human scaling law. Thus, a theoretical method to gain insight into vehicles' interaction with the road network considering micro-individuals' behaviours remains to be developed.

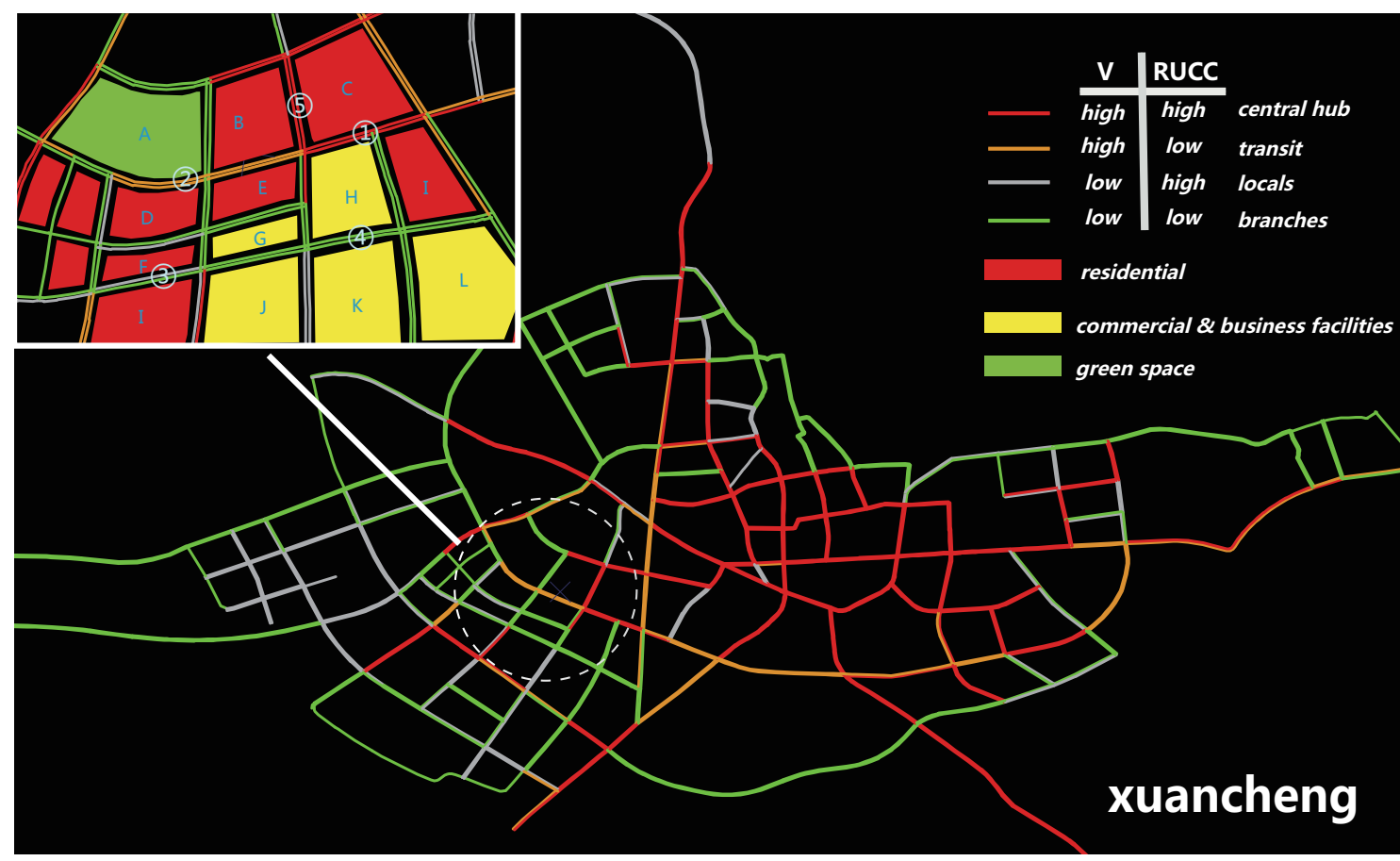

Figure 4. Types of road segments via classification of traffic flow and RUCC. (i) We grouped road segments into four types according to their traffic flow and RUCC. Both the larger proportion and smaller proportion were also cut by the average division point. The red symbols(central hub) represent roads with a larger traffic flow and RUCC. The dark yellow symbols(transits) represent those with larger parts of traffic flow and smaller parts of RUCC. The grey symbols(locals) are those with the smaller parts of traffic flow and larger parts of RUCC. These road segments show local roads' characteristics to serve the residents for everyday life and activities in non-hub areas. The road segments depicted in green have smaller parts of traffic flow and RUCC. They are always located in newly developed areas or connect high-grade roads(branches). (ii) The figure in the upper-left corner of the map is a microscopic view of road usage characteristics between land and interactive usage of vehicles. Different colours represent different land usage types: The red squares represent residential areas; yellow squares represent commercial or business areas, such as office buildings, banks, and shops; green squares represent green spaces, such as parks or lawns; circled numbers indicate road segments; and capital letters denote different blocks.

Given that RUCC characterises the usage diversity of road segments, it is interesting to combine it with traditional measures for comparison. Inspired by this, we conducted correlations tests between them. The results demonstrate that RUCC is slightly correlated with some aggregated indicators, such as traffic flow(see method: road segments classification Figure 5(a)). This corroborates our hypothesis that a road segment may have a larger traffic flow but a smaller road usage concentration coefficient(lower RUCC). Conceptually, traffic flow $V$ indicates road segments that many vehicles tend to use while RUCC represents the concentration degree that fixed but few vehicles like to use. To more exactly characterise a road's role, we classified segments into four groups with respect to their function according to traffic flow $V$ and road usage concentration coefficient $R U C C$ (as Figure 4 shows). We defined the central hubs(red symbols in Figure 4), which have higher $V$ and $R U C C$, and transits as the road segments with higher traffic flow but a lower road usage concentration coefficient(dark yellow symbols 
in Figure 4). Central hub road segments are frequently and diversely used by fixed vehicle sources while transits are frequently but less diversely used by fixed vehicles. The other two groups define some road segments with lower $V$ but higher RUCC as local roads(grey symbols in Figure 4), which serve residents' everyday lives and activities in non-hub areas, and branches(green symbols in Figure 4), which are located in newly developed areas or connect high-grade roads with lower $V$ and $R U C C$ (see specific method: road segments classification Figure 7(b)). As a result, a new morphological comparison of road segments could be obtained from the relationship between vehicles and road segment usage. We further explored the phenomenon of road usage from a micro perspective in combination with the land usage nature(as the upper-left figure shows in Figure 4). Both segments 1 and 2 comprised transit roads in the initial urban design, but since B and C blocks were developed for residential regions, the role of road segment 1 has transformed from transit to central hub roads while segment 2 maintains the characteristics of transits due to the planned green space of block A. Segment 3 shows the feature of local roads resulting from its weak connectivity from east to west and the residential properties of F block. It is used frequently by a few fixed vehicles but with low traffic compared to segments 1 and 2 . At the same grade, road segment 4 behaves totally differently because $\mathrm{G}, \mathrm{H}$, $\mathrm{J}, \mathrm{K}$, and $\mathrm{L}$ are commercial and business facilities; therefore, there are fewer fixed vehicles than in residential areas. This tells us that the nature and scale of the block which is closely connected to road segment determines the traffic demand of a given road segment, that is, the vehicles that are attracted and served. The characteristics of individual vehicle usage further shape the profile of road segments. In fact, the profile of road segments may change greatly in actual usage compared to the road function pictured by urban planners.

\section{Discussion}

The novel framework proposed in this study demonstrates that it is possible to obtain basic mobility regularities from large road networks benefiting from AVI data, which not only trace the major vehicles' sources of road segments, but also the road usage characteristics of urban roads. The first step of the method relied on the extraction of a vehicle mobility curve from the mobility network, and the average division point can be obtained given any spatial and temporal scale. The road usage concentration coefficient(RUCC) derived from the average division point then allowed us to characterise the functionality of roads, including their evolving profile in the city over the course of the day. These results constitute an expressive method to accurately manage vehicles and provide a quantitative picture of urban road usage.

The average division point we proposed differs from the Gini coefficient which is widely used to measure inequality; that is, the same Gini coefficient can have different Lorenz curves. Roughly speaking, it may be that the very largest individuals contribute a very large percentage of travel, but there may be other situations that are not unique (see Supplementary Figures S7 and S8). The Gini coefficient can capture the degree of inequality, but the inequality characteristics and distribution of the Lorenz curve are lost, while the average division point actually determines the trend, shape of the curve, and size of the Gini coefficient, which is the most original form of data distribution.

We also note here that several factors will inevitably affect the vehicle mobility curve that we ultimately want to extract, such as the density of the detector in the case of AVI data, data quality, or the spatial scale at which the mobility curve is constructed. The results may need to be further confirmed in another environment, but nevertheless, this study opens the door to use the identity of urban individual mobility data to grasp a city's macro operation rules and explore new transportation engineering theory, instead of traditional sampled-based surveys and aggregated approaches.

Our framework provides an efficient way to target the few but decisive vehicles of long-term urban road usage on the basis of AVI data. In this respect, it could be a particularly interesting direction for future research to apply the method to numerous contexts, such as congestion mitigation accurately associated with individuals, vehicle route strategies, personality management, etc. We can expect the selective strategies to be more effective and inexpensive than random governance strategies. We have presented a case study of congestion elimination using this novel framework as proof of our concept(see Supplementary Figure S10, Figure S11 and Figure S12, Figure S13 during the morning period). A certain road segment is long-term congested when the traffic flow exceeds its available capacity, particularly in the peak period. Taking a central road as an example, we found that the average traffic flow produced by vehicles in the three minorities $\left\{\left(x_{1}^{*}, y_{1}^{*}\right),\left(x_{2}^{*}, y_{2}^{*}\right),\left(x_{3}^{*}, y_{3}^{*}\right)\right\}$ respectively present a volume 4 , 10, and 19 times larger than the overall average. This corroborates the intuitive understanding that traffic flow is always caused by few vehicles, and reminds us to mitigate traffic flow by targeting the small proportion of vehicles with significantly more trips. We performed selective and random strategy separately for two samples of long-term congested road segments (including central road, Zhuangyuan South road and transit road, and Shaoting Middle road). For the two road segments, we reduced a certain number of vehicles, corresponding to the $\mathrm{m}$ total percentage of all vehicles using the road segment(m ranging from $0.1 \%$ to $1 \%$ for both road segments). Our selective strategy meant that the $\mathrm{m}$ percentages of vehicles were selected separately in the three targeted minorities $x_{1}^{*}, x_{2}^{*}, x_{3}^{*}$. A random strategy, in which vehicles are selected without combining the information of vehicles' contribution, was conducted for comparison(see Supplementary Figure S10 for the specific method). Taking the central Zhuangyuan South road as an example, the results are evident: when the selective strategy was used in the third minority $x_{3}^{*}$, reduced traffic flow reached 62,433 , corresponding to a $19.46 \%$ reduction in total traffic flow. However, when we used the 
random strategy to select vehicles, the corresponding reduced traffic flow was only 3378, approximately $1.05 \%$. This represents almost 18 times less reduction than the effect achieved by the selective strategy. Another interesting piece of information is provided as the number of minority layers continue to deepen (see Supplementary Figure S11). The traffic flow elimination of a central-type road with greater concentration is more obvious than that of a transit road, which means that when faced with a heavily congested road segment, we manage vehicles with less cost, but a more precise strategy and with better results. The underlying essence of the high efficiency is rooted in the above findings that the usage of each road segment can be attributed to few vehicles.

Another direction in future studies could be to investigate the time evolution of city or road usage patterns over several decades. We note here that this would comprise a generic method to depict the city forms and find abnormal usage patterns that have been described for so long by urban and traffic planners, and display how the city grows and varies with policies and development. It would also be interesting to apply the method to larger spatial scales. for example, to capture the openness level according to a comparison of RUCC between different cities. Similar to the Gini coefficient in economics, it may become a universal indicator for comparing urban openness and connectivity.

\section{Methods}

Data. The datasets for our study comprised AVI data, device layout data, road network data, and signal phase data from intersections. The data cover the entirety of Xuancheng city (approximately 562 square kilometres, 83.67\% of intersections have installed AVI devices in core areas) over a period of 1 year of records. The AVI data used in this research come from the electronic police system (see Supplementary 1.2) and are converted to virtual number. The electronic police bayonet (referred to as 'electric police bayonet') is installed at signal control intersections as a law enforcement tool to capture vehicles breaking the red light. It also records the information of each passing vehicle, and thus has a function of collecting traffic information. The AVI system differs from mobile or fixed detectors due to the following three features: 1) For each record, the system registers the vehicle's identity(license plate number); 2) it detects all vehicles rather than just low-occupancy operating vehicles such as taxis and buses; and 3) it distinguishes different lanes. The AVI system can achieve high definition capture due to its high detection and recognition rate. We verified our study data in contrast with real surveillance video by manual sampling to ascertain that the detection and recognition rates under normal lighting conditions were higher than $98 \%$ and $92 \%$ respectively, which could meet the needs of our research. For more specific data information, please refer to Supplementary note 1.3 .
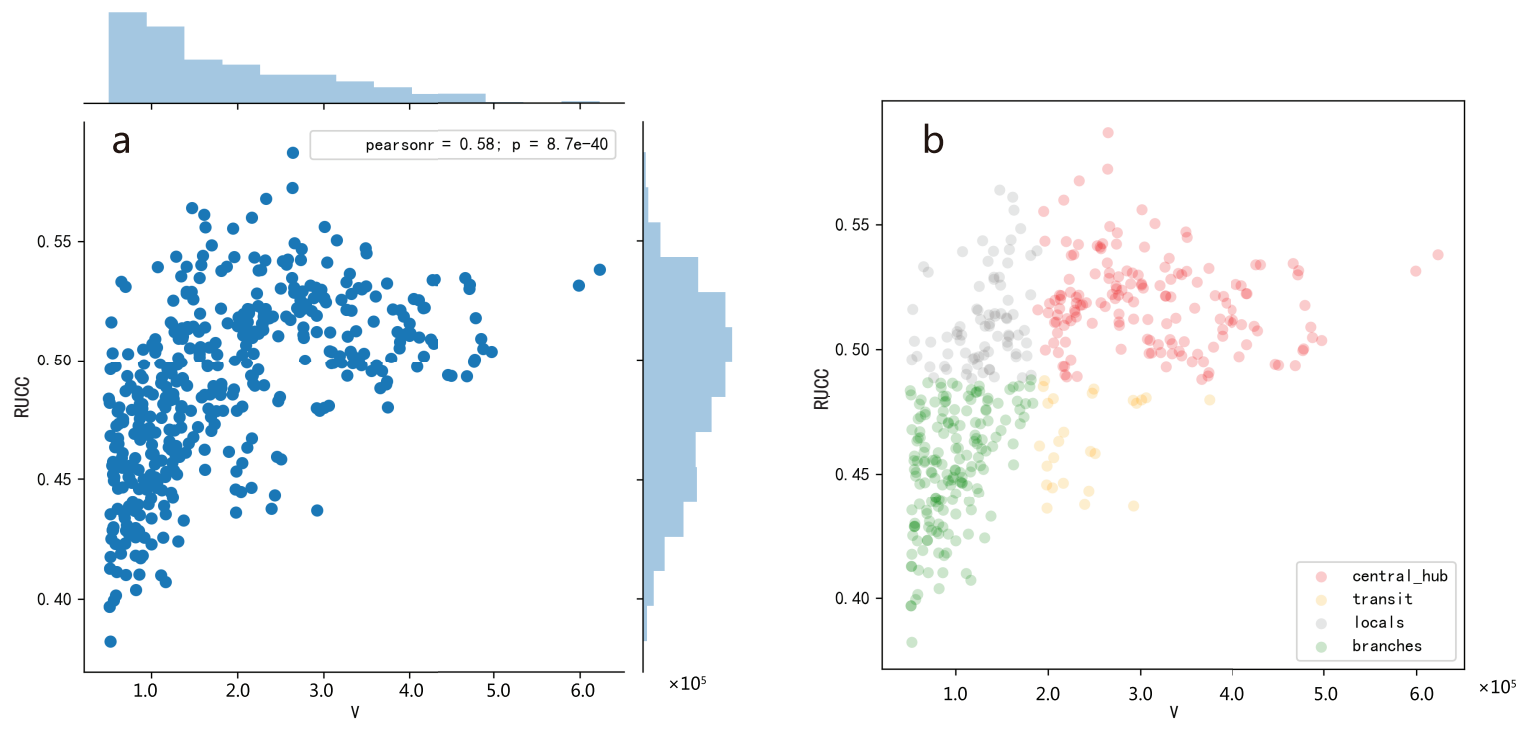

Figure 5. Grouping the road segments according to their traffic flow V and RUCC degree. (a) Road segment's traffic flow V is lowly correlated with its RUCC degree. (b) Categories of road segments via classification of traffic flow V and RUCC degree. The red symbols represent the road segments with higher traffic flow and RUCC. The yellow symbols represent those with higher traffic flow but lower RUCC. The grey symbols represent those with lower traffic flow but higher RUCC. The road segments depicted in green have lower traffic flow and RUCC.

reconstruction of vehicle mobility information from AVI data. To obtain more complete and rich vehicle mobility information such as trip time, we propose a hierarchical vehicle mobility information reconstruction framework that integrates AVI data 
and multi-source basic data(see supplementary note 2.1). We first organized AVI data into the detection sequence of vehicle individual $I_{v e h}=\left(I_{1}, I_{2}, \ldots I_{n}\right)$ (referred to as the 'AVI sequence'). Each record can be denoted as tuple $I_{n}=\left(v e h, t_{n}, \ldots p_{n}\right)$, including vehicle identity $v e h$, time stamp $t_{n}$, and location ID $p_{n}$. We then applied the vehicle's same travel identification model to divide each individual trip by the vehicle. The task of vehicle's same trip identification is that for a given vehicle detection sequence $I_{v e h}$, we need to determine whether any of two detection sequences $I_{i}$ and $I_{i+1}$ belong to the 'same trip' or 'two trips before and after'(see supplementary note 2.2 for specific process). Once we had obtained one trip of the vehicle, we performed a vehicle route repair based on road network topology and the Dijkstra shortest path algorithm. In so doing, we obtained the complete route of the vehicle trip and the time in and out of each road segment. See supplementary note 2.3 for the specific process.

road segments classification. Once the average division point of each road segment had been calculated based on vehicle mobility information extracted from AVI data, we obtained its traffic flow V and RUCC through $y^{*}-x^{*}$. As Figure 5(a) demonstrates, although the correlation coefficient for V and RUCC is 0.58 , we still found that road segments with similar traffic flow can differ greatly in vehicle mobility inequality degree RUCC. By the same token, for any given segment, we sorted the vehicles into decreasing rank, but here according to their V and RUCC, similar to Figure 1. We then obtained the average division point for $\mathrm{V}$ and RUCC, which is the criterion to distinguish between dominant and secondary vehicles. In so doing, road segments were divided into the following four categories in different colours.

\section{Data Availability}

The datasets generated during and/or analysed during the current study are available from the corresponding author on reasonable request

\section{References}

1. Louf, R. \& Barthelemy, M. How congestion shapes cities: from mobility patterns to scaling. Sci. Reports 4, 5561 (2014).

2. Batty, M. The new science of cities. Build. Res. Inf. 38, 123-126 (2013).

3. Ma, X., Liu, C., Wen, H., Wang, Y. \& Wu, Y. J. Understanding commuting patterns using transit smart card data. J. Transp. Geogr. 58, 135-145 (2017).

4. Kung, K. S., Greco, K., Sobolevsky, S. \& Ratti, C. Exploring universal patterns in human home-work commuting from mobile phone data. PLOS ONE 9 (2014).

5. Pei, T. et al. A new insight into land use classification based on aggregated mobile phone data. Int. J. Geogr. Inf. Sci. 28, 1988-2007 (2014).

6. Louail, T. et al. Uncovering the spatial structure of mobility networks. Nat. Commun. 6, 6007 (2015).

7. Louail, T. et al. From mobile phone data to the spatial structure of cities. Sci Rep 4, 5276 (2014).

8. Riccardo, G., Armando, B. \& Sandro, R. Towards a statistical physics of human mobility. Int. J. Mod. Phys. C 23, 1250061(2012).

9. Raja, J. et al. Understanding human mobility from twitter. (2015).

10. Chang, X., Shen, J., Lu, X. \& Huang, S. Statistical patterns of human mobility in emerging bicycle sharing systems. Plos One 13, e0193795 (2018).

11. Yan, X. Y., Wang, W. X., Gao, Z. Y. \& Lai, Y. C. Universal model of individual and population mobility on diverse spatial scales. Nat. Commun. 8, 1639 (2017).

12. Brockmann, ., D., Hufnagel, ., L. \& Geisel, ., T. The scaling laws of human travel. Nature 439, 462-465 (2006).

13. GonzÁlez, M. C., Hidalgo, C. A. \& BarabÁSi, A. Understanding individual human mobility patterns. Nature 453, 779-782 (2008).

14. Zipf, G. K. The p1p2/d hypothesis: On the intercity movement of persons. Am. Sociol. Rev. 11, 677-686 (1946).

15. Simini, F., González, M. C., Maritan, A. \& Barabási, A. L. A universal model for mobility and migration patterns. Nature 484, 96 (2012).

16. Filippo, S., Amos, M. \& Zoltán, N. Human mobility in a continuum approach. Plos One 8, e60069 (2013).

17. Ren, Y., Ercseyravasz, M., Wang, P., González, M. C. \& Toroczkai, Z. Predicting commuter flows in spatial networks using a radiation model based on temporal ranges. Nat. Commun. 5, 5347 (2014). 
18. Sobolevsky, S. et al. Delineating geographical regions with networks of human interactions in an extensive set of countries. PLOS ONE 8 (2013).

19. Jiang, B., Yin, J. \& Zhao, S. Characterizing the human mobility pattern in a large street network. Phys. Rev. E 80, 021136-021136 (2009).

20. Wen, T., Chin, W. \& Lai, P. Understanding the topological characteristics and flow complexity of urban traffic congestion. Phys. A-statistical Mech. Its Appl. 473, 166-177 (2017).

21. Gao, S., Wang, Y., Gao, Y. \& Liu, Y. Understanding urban traffic-flow characteristics: A rethinking of betweenness centrality:. Environ. Plan. B-planning Des. 40, 135-153 (2013).

22. Wang, J., Wei, D., He, K., Gong, H. \& Wang, P. Encapsulating urban traffic rhythms into road networks. Sci. Reports 4, 4141-4141 (2015).

23. Colak, S., Lima, A. \& Gonzalez, M. C. Understanding congested travel in urban areas. Nat. Commun. 7, 10793-10793 (2016).

24. Wang, P., Hunter, T., Bayen, A. M., Schechtner, K. \& González, M. C. Understanding road usage patterns in urban areas. Sci. Reports 2, 1001 (2012).

25. Schneider, C. M., Belik, V., Couronné, T., Smoreda, Z. \& González, M. C. Unravelling daily human mobility motifs. J. Royal Soc. Interface 10, 20130246 (2013).

26. Zhao, Z., Koutsopoulos, H. N. \& Zhao, J. Detecting pattern changes in individual travel behavior: A bayesian approach. Transp. Res. Part B-methodological 112, 73-88 (2018).

27. Noulas, A., Scellato, S., Lambiotte, R., Pontil, M. \& Mascolo, C. A tale of many cities: universal patterns in human urban mobility. (2011).

28. Gallotti, R., Bazzani, A., Rambaldi, S. \& Barthelemy, M. A stochastic model of randomly accelerated walkers for human mobility. Nat. Commun. 7, 12600 (2016).

29. Pappalardo, L. et al. Returners and explorers dichotomy in human mobility. Nat. Commun. 6, 8166-8166 (2015).

30. Pappalardo, L., Rinzivillo, S., Qu, Z., Pedreschi, D. \& Giannotti, F. Understanding the patterns of car travel. Eur. Phys. J. Special Top. 215, 61-73 (2013).

31. Goodchild, M. F. \& Janelle, D. G. The city around the clock: Space-time patterns of urban ecological structure:. Environ. Plan. A 16, 807-820 (1984).

32. Nechet, F. L. Urban spatial structure, daily mobility and energy consumption: a study of 34 european cities. Cybergeo: Eur. J. Geogr. (2012).

33. Jiang, B. A topological pattern of urban street networks: Universality and peculiarity. Phys. A-statistical Mech. Its Appl. 384, 647-655 (2007).

34. Foley, D. L. The daily movement of population into central business districts. Am. Sociol. Rev. 17, 538 (1952).

\section{Acknowledgements}

The authors acknowledge Guangdong Fundway Technology CO., Ltd for the data sets and Editage (www.editage.cn) for English language editing. This research is funded by the National Natural Science Foundation of China (No. U1611461).

\section{Author contributions statement}

B.Z. and Z.Y, Z.C.H designed the study; B.Z. analyzed the data and wrote the manuscript; M.H, K.Y.C, L.K.L coordinated the study and interpreted the results. All authors read, commented, and approved the submitted manuscript.

\section{Additional information}

Competing Interests: Te authors declare no competing interests. 